\title{
COPTOPSYLLIDAE (SIPHONAPTERA) AFRICAINES : REPARTITION, MORPHOLOGIE, STATUT TAXONOMIQUE ET RELATIONS PHYLÉTIQUES \\ AVEC LES AUTRES REPRÉSENTANTS DE LA FAMILLE
}

\author{
H. LAUNAY* et J.-C. BEAUCOURNU***
}

\begin{abstract}
RÉSUMÉ. La distribution géographique des Coptopsyllidae africaines (Siphonaptera) est donnce, leur morphologie est précisée. Coptopsylla joannae Lewis, 1964 est mise en synonymie avec C. africana Wagner, 1932 et le genre Neocoptopsylla Wagner, 1932 est revalidé. Des caractères pour une clé des mâles des 22 espèces et sous-espèces paléarctiques sont proposés.
\end{abstract}

Mots-clés : Coptopsyllidae. Neocoptopsylla wassiliewi. Coptopsylla africana. Afrique.

African coptopsyllids (Siphonaptera): geographical distribution, morphology, taxonomical status and phyletic relationships with the other members of the family.

SUMMARY. Geographical distribution and morphology of African fleas belonging to the family Coptopsyllidae (Siphonaplera) are given. Coptopsylla joannae Lewis, 1964 is a synonym of C. a/ricana Wagner, 1932 and the genus Neocoplopsylla Wagner, 1932 is valid. Characters for a key to the males of the 22 palearctic species and subspecies are provided.

Key-uords : Coplosyllidae. Neocoptopsylla wassiliewi. Coptopsylla africana. Africa.

La famille des Coptopsyllidae Wagner, 1928, regroupait 23 taxa dans le seul genre Coptopsylla Jordan et Rothschild, 1908 (voir Lewis, 1974). Sur le plan phylétique, elles se placent, à l'intérieur des Hystrichopsylloüdea, à côté des Hystrichopsyllidae sensu Smit, 1982 (genres Hystrichopsylla, Ctenoparia, Typhloceras, Atyphloceras et Macropsylla) seule famille, avec elle, à présenter deux spermathèques chez la femelle.

Les Coptopsyllidae sont toutes des parasites de Gerbillidés, rongeurs paléarctiques adaptés à la vie dans les steppes et les zones arides et désertiques d'Afrique et d'Asie.

* Instilut de Parasitoloyie de l'Ouesl, Facullé de .Médecine, avenue du P'rofesseur-Léon-Bernard, F 35043 Rennes Cedex.

** Laboraloire de Parasilologie (Entomologie médicale), Faculté de Médecine, Rennes.

Accepté le 15 ociobre 1986. 
La répartition actuellement connue de ces ectoparasites est restreinte par rapport à celle de leurs hôtes puisqu'elle se limite à la partie sud des sous-régions méditerranéenne et sibérienne, c'est-à-dire du Maroc à l'Afghanistan.

En Afrique, les Coptopsyllidae n'ont été trouvées qu'au nord du Sahara et sont représentées par Coptopsylla wassiliewi (Wagner, 1932) (zones semi-arides', type steppes à alfa du Maroc, d'Algérie et de Tunisie) et Coptopsylla africana Wagner, 1932, qui semble plus xérophile (zones désertiques à graminées et buissons du sud Tunisien).

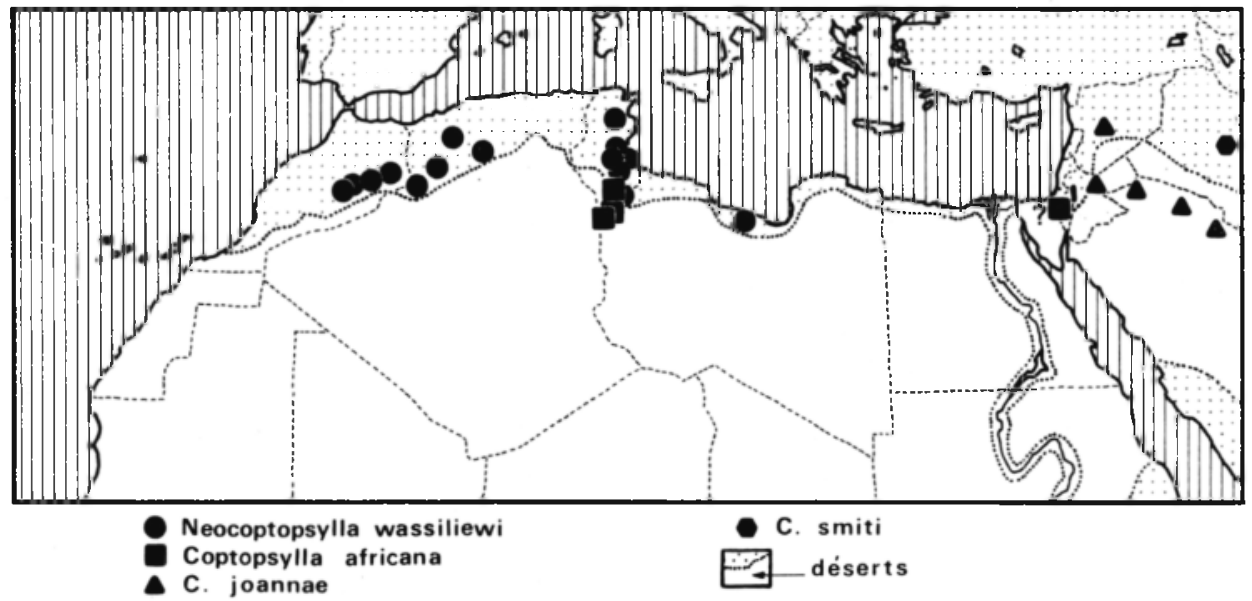

Fig. 1. -- Stations connues des Coptopsyllidae dans la région considérée.

Nous avons eu la possibilité d'examiner deux femelles (dont l'holotype) et le seul mâle connu de C. africana ${ }^{2}$, ce qui nous a permis de revoir certains points de morphologie mal précisés ou négligés par Wagner lors de la description princeps. Ceci nous a semblé d'autant plus utile que ces manques ou erreurs ont été unanimement repris par tous les auteurs qui se sont attachés à cette famille (par exemple, Hopkins et Rothschild, 1956 ; Lewis, 1964).

D'autre part, nous avons réétudié les wassiliewi qui se trouvent dans nos collections, et, en replaçant cette espèce par rapport aux autres Coplopsyllidae, il nous a semblé nécessaire de revalider le genre Neocoptopsylla Wagner, 1932, dont elle est l'espèce type (et unique).

1. Contrairement à la répartition totalement crronée de Jameson, 1985, qui place $N^{\circ}$. wassiliewi dans le désert (fig. 2, p. 869).

2. Grace à l'obligeance du Docteur F*. Rodhain, Unilé d'F́cologie virale, Instilut Pasteur de Paris (France); nous le remercions très vivement. 

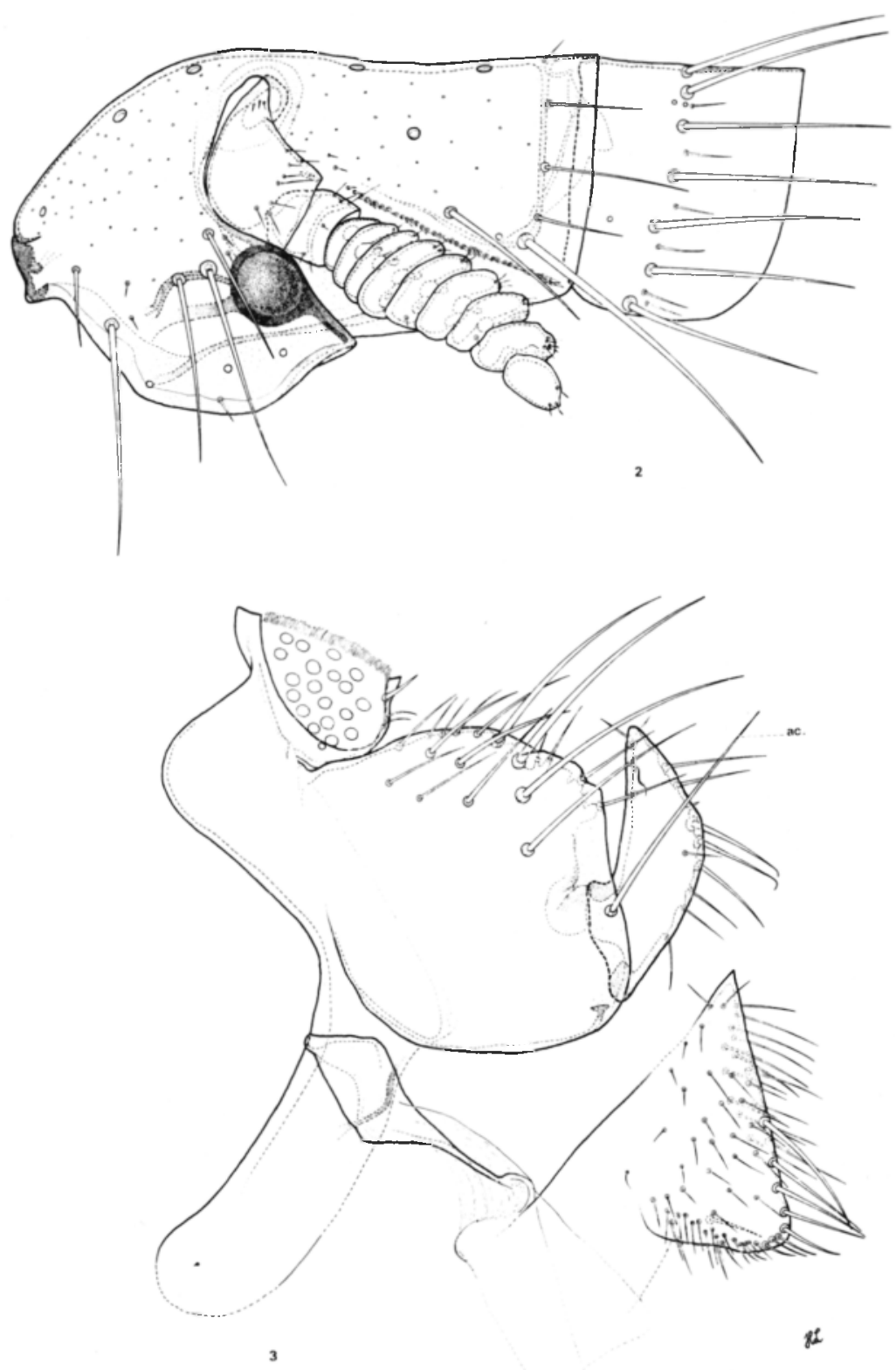

Fì. 2 el 3. Coptopsylla africana, mâle allotype, Bir Pistor ('Tunisie).

Fig. 2 : Capsule céphalique et pronotum.

Fig. 3 : Segment IX, ac. = soie acétabulaire. 


\section{I - Généralités : les caractères morphologiques à retenir pour la diagnose des Coptopsyllidae}

Nous avons revu les descriptions des Coptopsyllidae connues à ce jour et il nous a semblé utile, chez cette famille relativement homogène, de redéfinir les caractéristiques morphologiques essentielles pour la détermination des différents taxa. Nous ne reprendrons donc pas ici les critères qui définissent la famille.

\section{1 - La capsule céphalique (POUR les deuX SeXes) \\ $a$ - LE CLYPEUS}

Cette sclérification qui se situe à l'extrémité antérieure de la tête, au-dessus de l'angle oral, est particulièrement bien marquée chez les Coptopsyllidae. Sa forme est importante et nous verrons qu'elle constitue un des critères qui permet de séparer le genre Neocoptopsylla (fig. 16) du genre Coptopsylla (fig. 2).

\section{$b$ - Les Soies de la partie pré-ANTENNaire}

Ces soies se répartissent en deux rangées (fig. 2) :

— une rangée frontale qui comprend (pour les espèces actuellement connues) de 0 à 2 soies; dans ce cas, il y a une soie assez courte juste en arrière du clypeus et une soie, souvent plus longue, nettement au-dessus de l'œil, près du bord de la fosse antennaire;

- une rangée oculaire qui comprend (pour les espèces actuellement connues) 3 soies et qui se projette généralement au niveau de l'arc du tentorium.

La soie la plus antérieure est située près du bord de la capsule céphalique, la soie la plus postérieure est située près de l'œil.

Des petites soies très courtes sont en nombre variable et n'ont pas d'intérêt taxonomique.

\section{2 - LES SOIES LATÉRALES DU TARSE V (POUR LES DEUX SEXES)}

Les grosses soies latérales du cinquième segment tarsal sont en nombre constant, soit normalement six paires pour toutes les Coptopsyllidae connues (fig. 11) sauf chez wassiliewi où il y a cinq paires de soies (quelquefois quatre pour la patte postérieure) (fig. 19).

\section{3 - LE SEGMENT IX DU MALE}

$a$ - LE TERGITE

Schématiquement, le basimère ( $P$ 1) s'inscrit dans une figure quadrangulaire (fig. 3). Ia forme de son contour va dépendre essentiellement de l'absence ou de la 

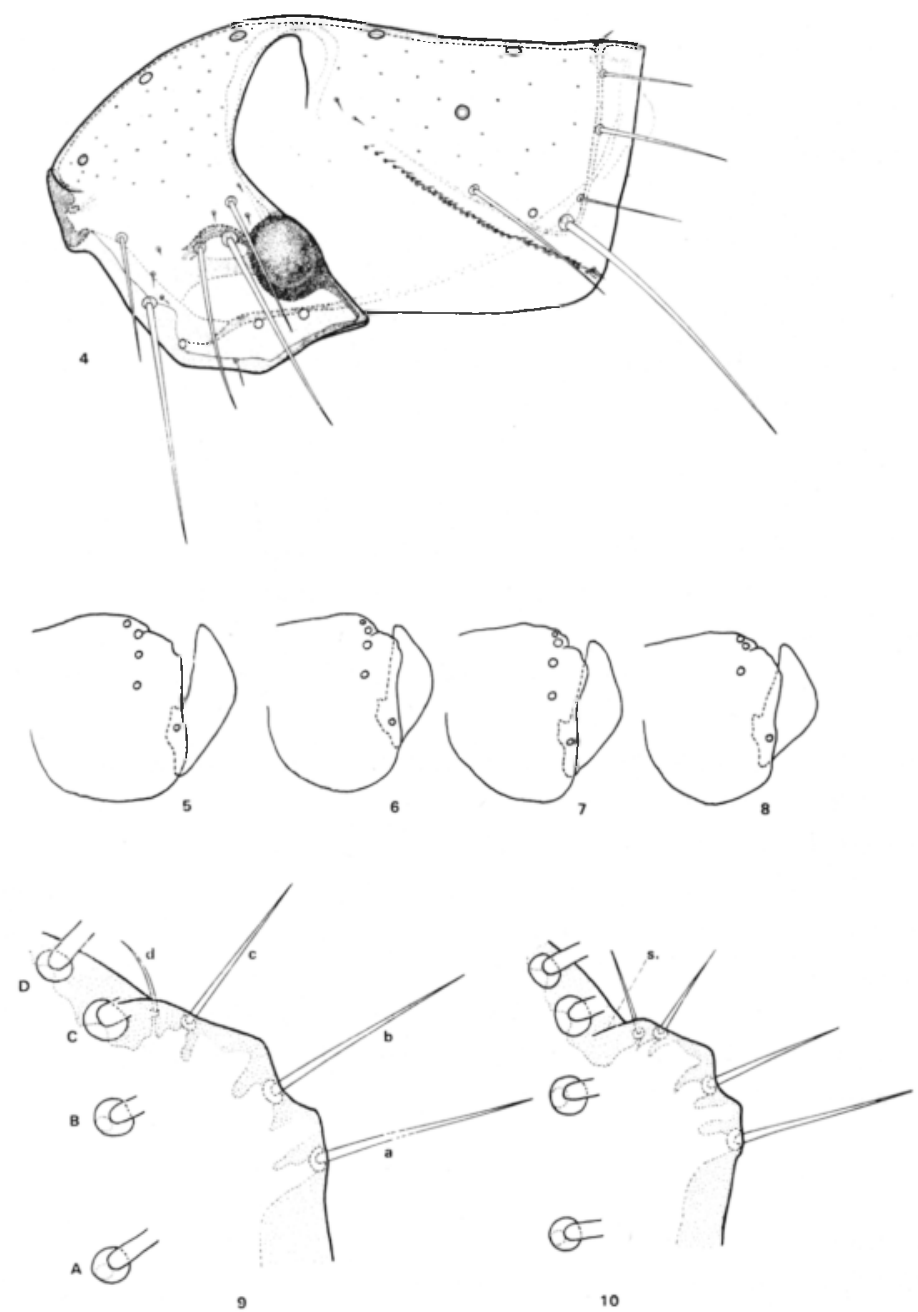

Fig. 4. - Coplopsylla joannae, mâle, Palmyra (Syrie), capsule céphalique (antenne omise).

Fig. 5 à 8 . - Variations du tergite IX.

Fig. 5 : Coptopsylla africana, allotype, Bir Pistor (Tunisie).

Fig. 6 à 8 : Coptopsylla joannae, Palmyra (Syrie).

Fig. 9. - Coptopsylla africana, allotype, Bir Pistor (Tunisie), angle postéro-supérieur du basimère.

Fig. 10. - Coptopsylla joannae, Palmyra (Syrie), angle postéro-supérieur du basimère, s. = sinus. 
présence et de l'importance d'un sinus qui va déterminer deux lobes, de la hauteur relative de ceux-ci et de leur écartement.

La sétation du basimère comprend :

- sur le bord postéro-inférieur : la soie acétabulaire (fig. 3, ac.). La projection de l'insertion de cette soie par rapport à l'acetabulum (qui correspond à la base du télomère) est un critère taxonomique ;

- au niveau de l'angle supérieur du bord postérieur : une série de quatre soies courtes ( $f g .9, a, b, c$ et $d$ ) implantées sur la face interne. Plus en avant, une série de quatre soies longues ( $f$ ig. $9, A, B, C$ et $D$ ) qui sont implantées sur la face externe du basimère. En avant de ces grandes soies, et jusqu'au bord dorsal, toute une série de soies de taille et de nombre variables sont sans intérêt taxonomique.

Quand il existe un sinus, qui peut être réduit à une simple incisure (fig. 10, s.), celui-ci se trouve en arrière des grandes soies A, B, C, D qui borderont par conséquent le lobe antérieur ( = processus basimeris dorsalis) et en avant des petites soies $a, b, c, d$, qui se situeront à l'apex du lobe postérieur (= processus basimeris ventralis). Chez Coptopsylla arax Isayeva-Gurvich, 1950, ce sinus est particulièrement large et profond puisqu'il descend jusqu'au niveau de l'insertion de la soie acétabulaire.

Une ou plusieurs des soies que nous venons de mentionner peuvent manquer : c'est, par exemple, le cas de la soie acétabulaire chez C. l. lamellifer (Wagner, 1895) et $C$. caucasica Isayeva-Gurvich, 1950.

Le télomère a une forme simple, arquée. Le bord postérieur est convexe, le bord antérieur est droit ou concave, souvent marqué par une protubérance qui trouve son vis-à-vis sous la forme d'une fossette située sur le bord postérieur du basimère, au niveau des petites soies $a, b, c, d$ (fig. 9). Le rapport hauteur sur largeur du télomère, la forme de son apex (pointu, recourbé, arrondi...) sont des critères de détermination.

\section{$b$ - LE STERNITE}

Il y a une tendance à la réduction du sternite IX chez les Coptopsyllidae et à sa soudure avec les lobes latéraux du phallosome.

Seule la partie apicale du bras distal est bien individualisée et utilisable pour la systématique.

\section{4 - LES SOIES ANTESENSILIALES ET LES SPERMATHĖQUES CHEZ LA FEMELLE}

La longueur relative des deux soies antésensiliales est un critère taxonomique classique, de même que la forme des spermathèques. Il faut tout de même noter que chez les Coptopsyllidae la diagnose au niveau spécifique n'est pas toujours possible en se référant uniquement à la morphologie des spermathèques. Il y a d'ailleurs une variabilité de cette morphologie pour une espèce donnée, en particulier pour la plus petite des deux spermathèques. 

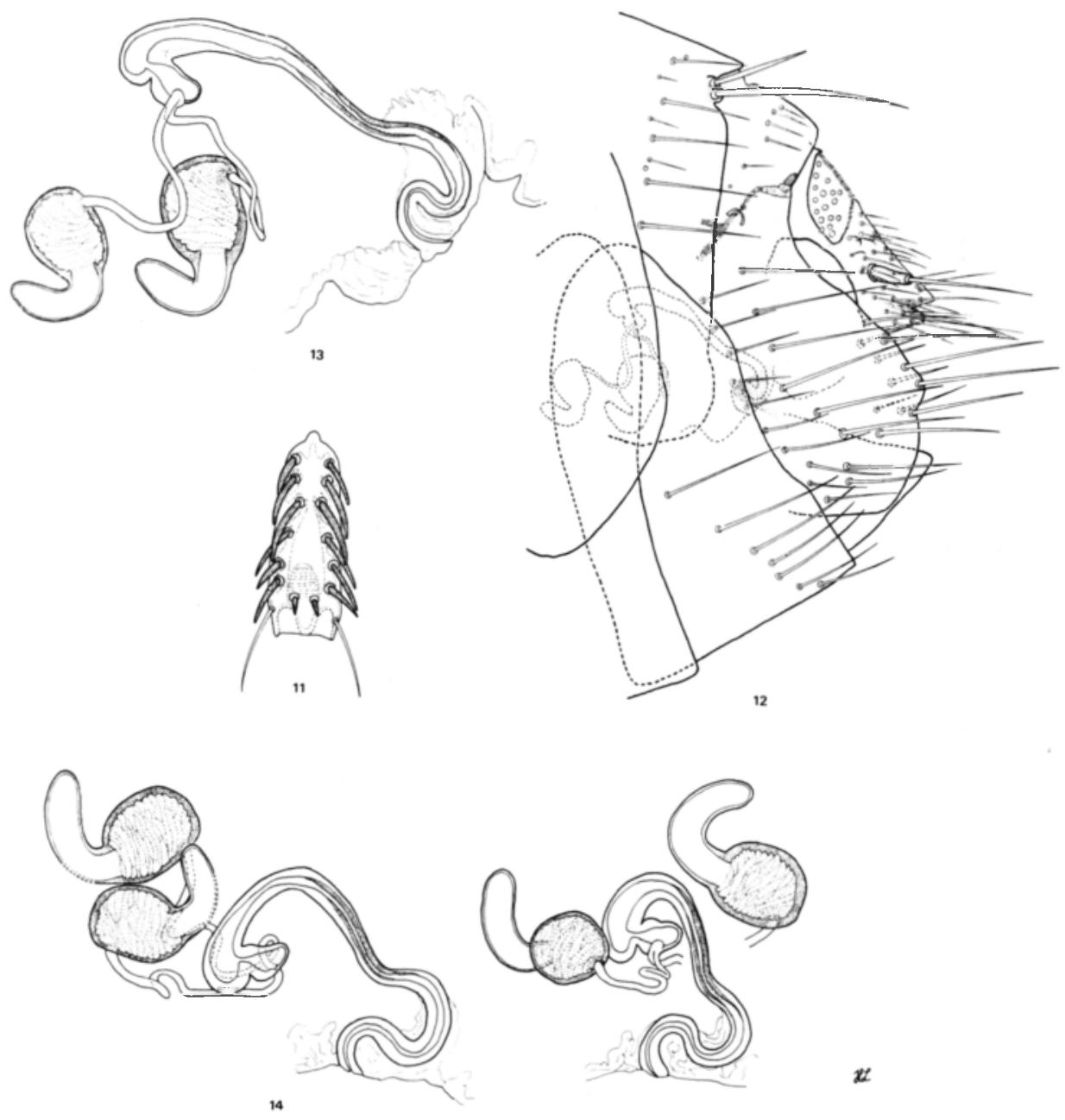

15

Fig. 11 et 12. - Coptopsylla africana, femelle, Fort-Saint (Tunisie).

Fig. 11 : Tarse 5 de la patte postérieure.

Fig. 12 : Segments terminaux.

Fig. 13 à 15. - Conduits génitaux et spermathèques.

Fig. 13 : Coptopsylla africana, Fort-Saint (Tunisie).

Fig. 14 : Coptopsylla africana, holotype, Fort-Saint (Tunisie).

Fig. 15 : Coptopsylla joannae, Palmyra (Syrie). 


\section{II - Les Coptopsyllidae d'Afrique}

\section{1 - COPTOPSYLLA AFRICANA WAGNER, 1932}

Coptopsylla africana Wagner, 1932 a été décrite (1932a, p. 267-268, fig. 1) à partir d'une seule femelle, holotype, récoltée par Wassilieff sur Gerbillus sp., en novembre 1930, dans l'extrême sud tunisien, à Fort-Saint (Wagner et Wassilieff, 1932, p. 463).

Lors d'une seconde mission dans la même région en janvier 1932, Wassilieff récolta à nouveau cette espèce : 1 mâle sur Meriones schousboei ( = Meriones libycus) à Bir Pistor et 16 femelles sur des Psammomys roudairei (= Psammomys obesus) ou dans leurs nids à Fort-Saint, Djenneïen, M'Chiguig (Wassilieff, 1932, p. 330) (15 femelles d'après Wagner, 1932b, p. 170).

Le mâle (qui doit être désigné comme un néallotype) a été également décrit par Wagner (1932b, p. 169-170, fig. 2)

Synonyme : Coptopsylla joannae Lewis, 1964 (cf. discussion).

Répantition : C. africana n'a jamais été recapturée en Afrique du Nord depuis les expéditions de Wassilieff dans le sud tunisien (cette zone n'a jamais été réétudiée depuis). Elle est donc connue de Fort-Saint, Bir Pistor, M'Chiguig, Djenneïn (Tunisie).

Par ailleurs, une femelle, capturée sur un Gerbillus dasyurus à Nahal Nafha en Israël, a été attribuée à C. africana (Theodor et Costa, 1967, p. 64). Cette détermination demanderait à être confirmée par la capture et l'examen de mâles.

C. joannae est connue du nord de l'Arabie Saoudite, de Syrie (Lewis, 1964a, 1964b) et de Jordanie (Lewis, 1966).

Iconographie : Wagner, 1932a : fig. 1, p. 267, femelle holotype, tergite VIII, sternites VII et VIII, spermathèques et ducti; Wagner, 1932b : fig. 1, p. 168, femelle, capsule céphalique, fig. 2, p. 169, mâle néallotype, tergite et sternite IX ; Wagner et Wassilieff, 1932 : fig. 43, p. 461, femelle, tarse 5 de la patte postérieure, fig. 44, p. 462, femelle, capsule céphalique.

MORPHOLOGIE :

- Tête (fig. 2).

Clypeus typique des Coptopsylla, bien développé et sclérifié, sa hauteur est égale au diamètre de l'œil, plat sur sa face antérieure, bilobé en arrière. Le sillon céphalique antérieur de la capsule céphalique se projette au niveau du sommet du clypeus.

La rangée oculaire comprend 3 soies, la rangée frontale comprend 2 soies.

- Le segment IX du mâle (fig. 3 et 9 ).

Le basimère possède un sinus mais celui-ci est discret et se réduit à une incisure peu profonde qui se projette, chez le seul mâle connu, au niveau de la 3 e grande soie externe (soie C, fig. 9). Le processus basimeris ventralis présente quatre soies marginales sur sa face interne et une légère dépression (au niveau de la soie $b$, fig. 9) en vis-à-vis d'une protubérance sur la face externe du télomère. 
La soie acétabulaire se projette au niveau de l'acelabulum.

Bord antérieur du télomère droit, bord postérieur convexe, les soies marginales sont sur la face interne ; l'apex forme un angle aigu, cependant le bout n'est pas pointu mais mousse; environ 3 fois plus haut que large.

La partie apicale du bras distal du sternite est bien visible, avec de nombreuses soies sur les faces interne et externe, elle a une forme triangulaire avec un apex pointu et un angle postéro-inférieur arrondi ; quelques grandes soies marginales externes au-dessus de cet angle.

- Les soies antésensiliales et les conduits génitaux chez la femelle (fig. 12, 13 et 14).

Nous avons pu vérifier, sur les deux exemplaires en notre possession (dont l'holotype) que la soie antésensiliale supérieure fait environ la moitié de la longueur de la soie inférieure, en opposition à la description de Wagner (1932a) qui indique qu'elles sont de longueur presque égale : "... welche beinahe gleich lang sind " (p. 268). Cette observation erronée est reprise par Hopkins et Rothschild (1956) tant dans la clé de détermination des Coplopsyllidae (p. 57) que dans la description de C. africana (p. 65).

Le ductus bursae est long, flexueux, à trois courbures, la partie médiane, presque rectiligne, est renforcée par une sclérification tant sur ses faces antérieure que postérieure.

La perula est peu développée, les ducti spermathecae, courts, sortent d'une dilatation en forme d'ampoule.

La spermathèque gauche est sensiblement plus petite que la droite, mais de même morphologie. Bulga ovoïde, aussi longue que haute, hilla de la même longueur que la bulga, émergeant sans transition de celle-ci.

\section{2 - NEOCOPTOPSYLLA WASSILIEWI WAGNER, 1932}

Neocoptopsylla wassiliewi Wagner, 1932, g. n., sp. n., a été décrite (1932b, p. 171-175, fig. $3,4,5,6$ et 7) à partir de 6 mâles et 5 femelles récoltées par Wassilieff en novembre 1931 (Wagner, 1932b, p. 175) sur Meriones shawi (Wagner et Wassilieff, 1932, p. 462) ou dans ses nids, à Bir Mellah (Tunisie).

Le genre Neocoplopsylla a été descendu au rang de sous-genre par Ioff et Tiflov (1934) puis mis en synonymie, à notre avis abusivement, avec le genre Coptopsylla par Hopkins (1952).

Synonymie : Coptopsylla (Neocoptospylla) wassiliewi (Wagner, 1932), Ioff et Tiflov, 1934; Coptopsylla wassiliewi (Wagner, 1932), Hopkins, 1952.

Répartition : contrairement à $C$. africana, N. wassiliewi a fréquemment été retrouvée en Afrique; elle est maintenant connue, outre la Tunisie, du Maroc, d'Algérie et de Libye.

Maroc : Province de Ksar-es-Souk (Hastriter et Tipton, 1975).

Algérie : Béni-Ounif de Figuig (Hopkins et Rothschild, 1956), Brezina, Laghouat, Aïn Sefra (Beaucournu et Kowalski, 1985). 
Tunisie : Bir Mellah, I)jenneien (Wagner et Wassilieff, 1933), Tamezret, Gabès, Tatahouine (Beaucournu et Hellal, 1977), Médénine (Beaucournu et coll., 1981).

Libye : Nofilia (Cooreman, 1973).

Iconographie : Wagner, 1932b : fig. 5, tergite IX du mâle lectotype (selon Smit et Wright, 1965 ; ces auteurs indiquent en outre que les autres spécimens de la série de description, soit 5 mâles et 5 femelles doivent être considérés comme des paralectotypes), fig. 3 , p. 170 , mâle paralectotype, tarse 5 de la patte antérieure, fig. 4 , p. 171 , mâle paralectotype, tarse 5 de la patte postérieure, fig. 6 , p. 173, femelle paralectotype, capsule céphalique, fig. 7, p. 174, femelle paralectotype, tergite VIII, sternites VII et VIII ; Wagner et Wassilieff : 1932, fig. 42, p. 461, femelle, capsule céphalique ; Cooreman, 1973 : p. 3, fig. 1, femelle, capsule cépha-
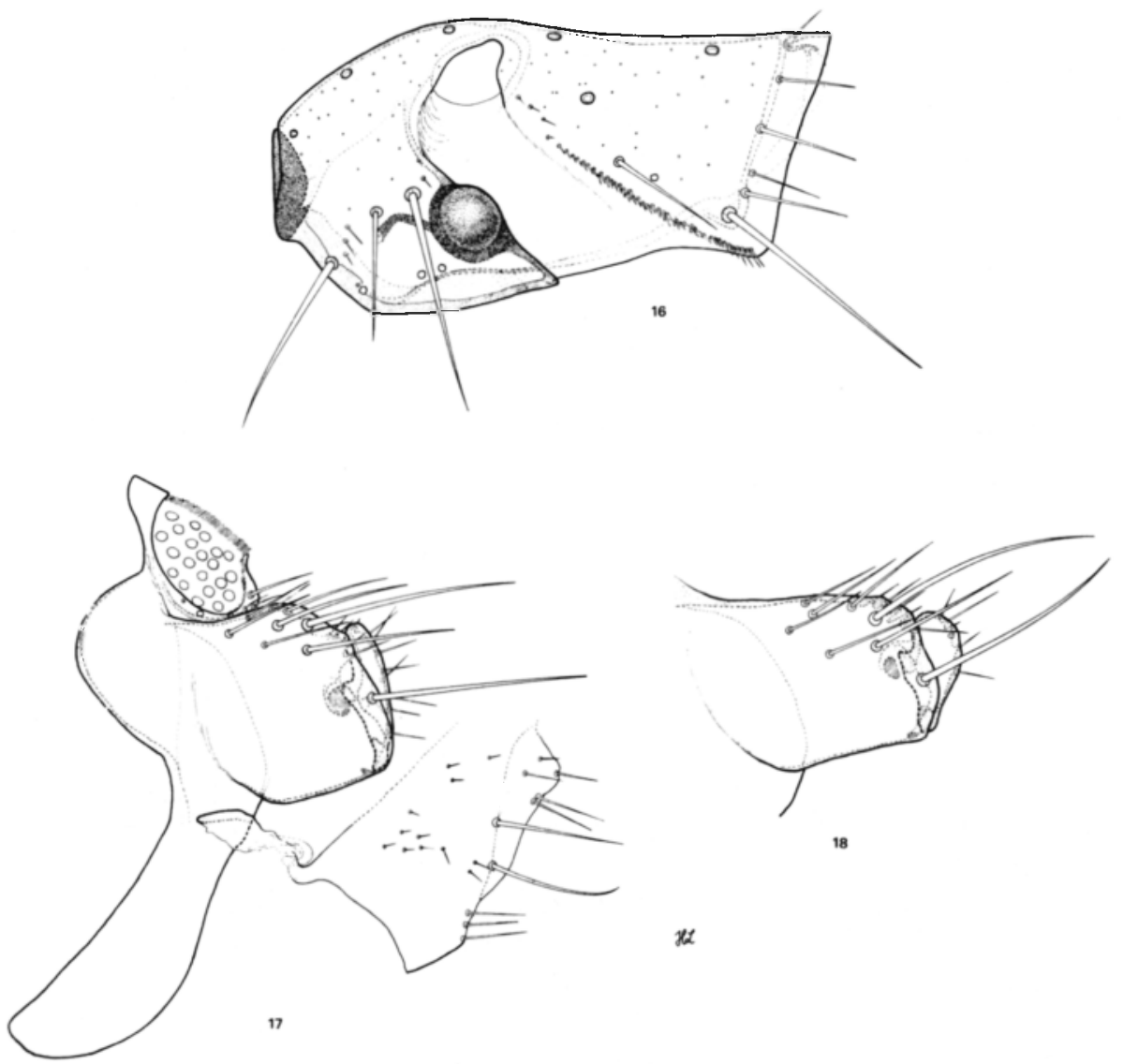

FIg. 16 à 18. - Neocoplopsylla wassiliewi, mâle.

Fig. 16 : Aïn Sefra (Algérie), capsule céphalique (antenne omise).

Fig. 17 : Foum Tatahouine (Tunisie), segment IX.

Fig. 18 : Aïn Sefra (Algérje), tergite IX d'un spécimen parasité par des Tylenchides. 
lique, fig. 2, mâle, tergite IX (cet exemplaire est anormal : télomère élargi, apodème tronqué, manubrium régressé ; il était très probablement parasité par des tylenchides et partiellement castré, ce qui est fréquent chez $N$. wassiliewi (Beaucournu et Hellal, 1977)), p. 4, fig. 3 , mâle, tarse 5 de la patte II, fig. 4 , mâle, tarse 5 de la patte III, fig. 5, femelle, tergite et sternite VIII, fig. 6 , femelle, spermathèques.

MORPHOLOGIE :

- Tête (fig. 16).

Clypeus très massif, protubérant, plat sur sa face antérieure, convexe sur sa face postérieure, sa hauteur est égale au double du diamètre de l'œil. Le sillon horizontal antérieur de la capsule céphalique est peu visible car il se projette au niveau de la partie médiane du clypeus.

La rangée oculaire comprend 3 soies, la rangée frontale est absente.

- Le segment IX du mâle (fig. 17).

Le segment IX de wassiliewi frappe par sa petite taille (cf. les figures 3 et 17 qui sont à la même échelle) pour une puce qui a des dimensions " normales ".

Le basimère ne présente pas de sinus. La soie acétabulaire se projette au niveau de l'acetabulum.

Le télomère est petit, les bords sont sub-parallèles, l'apex est très arrondi.

Le bras distal du sternite IX est particulièrement peu visible chez wassiliewi, il se confond avec les parois latérales du phallosome. L'apex est indistinct; un petit lobe ventral est souligné par une suture qui porte 2 ou 3 grandes soies.

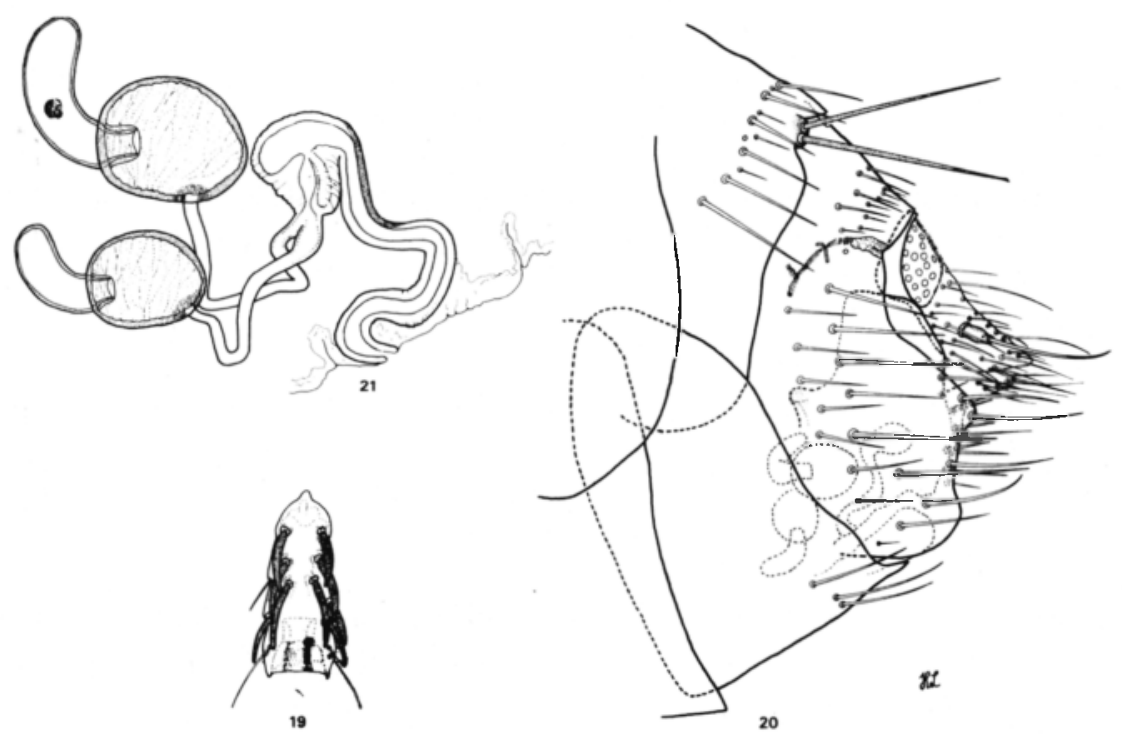

FIG. 19 à 21. - Neocoptopsylla wassiliewi, femelle, Foum Tatahouine (Tunisie).

Fig. 19 : Tarse 5 de la patte postérieure.

Fig. 20 : Segments terminaux.

Fig. 21 : Conduits génitaux et spermathèques. 

et 22).

-- Les soies antésensiliales et les conduits génitaux chez la femelle (fig. 21

Les soies antésensiliales supérieure et inférieure sont de la même longueur.

Le ductus bursae est du type C. africana en plus trapu. L'ampoule qui débouche de la perula est massive, à parois épaisses, formant un puissant sphincter.

Les ducti spermathecae, courts, sont légèrement dilatés à leur base.

Les spermathèques sont dissymétriques, la gauche étant toujours plus petite que la droite. La bulga est ovoïde, légèrement plus longue que haute. La hilla, sensiblement de même longueur que la bulga, est profondément enchâssée dans celle-ci.

\section{III - Discussion}

\section{1 - COPTOPSYLLA AFRICANA ET C. JOANNAE}

Nous avons pu comparer $C$. africana avec 3 couples de $C$. joannae Lewis, $1964^{3}$.

Lors de sa description, $C$. joannae a été rapprochée de C. bairamaliensis Wagner, 1928 dont elle diffère pour l'essentiel par les soies du corps moins épaisses. Il est vraisemblable que $C$. africana avait été écartée car elle était décrite comme ne possédant pas de sinus au niveau de son basimère, critère utilisé d'emblée pour la diagnose des mâles de Coptopsyllidae dans les clés de détermination existantes.

Nous avons vu que le mâle "néallotype " de C. africana présente une incisure sur le basimère, discrète mais tout à fait comparable à celle de $C$. joannae $($ fig. 9,10$)$; l'ensemble du tergite IX de ce mâle unique entre d'ailleurs dans les variations des exemplaires de $C$. joannae que nous avons pu examiner (fig. 5, 6, 7 et 8). Il en est de même du sternite IX, de tous les autres segments et de leur sétation et ceci pour les deux sexes, de la capsule céphalique (fig. 2 et 4), des segments terminaux femelles, des soies antésensiliales, des conduits génitaux et des spermathèques (fig. 13, 14 et 15).

Cette similitude de morphologie nous amène à proposer de mettre en synonymie $C$. africana et $C$. joannae syN. Nov.

Sur le plan écologique rien ne s'y oppose puisque, à l'image de C. africana, toutes les captures de $C$. joannae ont été effectuées en zone désertique (moins de $100 \mathrm{~mm}$ de pluies par an, fig. 1).

C. africana serait finalement une espèce inféodée aux Gerbillidés xérophiles, et biogéographiquement répartie dans les zones désertiques ou prédésertiques depuis le sud maghrébin jusqu'à la péninsule arabique.

3. Qui nous ont été gracieusement donnés par le Professeur R. E. Lewis, Department of Entomology, Iowa states University (U.S. A.) ; nous lui exprimons toute notre gratitude. 


\section{2 - LE GENRE NEOCOPTOPSYLLA WAGNER, 1932, GEN. REVOC.}

Il nous semble indispensable de revalider le genre Neocoptopsylla mis en synonymie avec Coptopsylla par Hopkins (1952).

En effet, wassiliewi qui est l'espèce-type et unique de ce genre présente des caractères singuliers à l'intérieur des Coptopsyllidae, et qui se situent au moins à un rang générique :

- pour les deux sexes : l'absence de rangée de soies frontales, le clypeus massif et convexe en arrière, cinq paires de soies plantaires pour le dernier tarse de la patte postérieure;

- pour la femelle : deux soies antésensiliales de la même longueur et surtout les spermathèques qui présentent une hilla profondément enchâssée dans la bulga.

Enfin, nous pouvons rappeler chez le mâle la petite taille du segment IX, le télomère trapu et arrondi à son apex et le sternite IX vestigial.

\section{3 - LES GROUPES D'ESPËCES DANS LE GENRE COPTOPSYLLA}

Nous avons précédemment indiqué que les clés de détermination des Coptopsylla étaient basées d'emblée sur la morphologie du sinus du basimère pour les mâles, ce qui amène des difficultés; pour les femelles seule la morphologie des spermathèques est prise en compte ce qui crée des problèmes encore plus grands.

Il nous semble plus facile, et plus logique phylétiquement, d'utiliser d'autres critères qui permettent d'emblée de séparer des groupes d'espèces et ceci d'une façon claire : la sétation de la capsule céphalique, la position de la soie acétabulaire.

Au niveau générique :

- pas de soies dans la rangée frontale : g. Neocoptopsylla,

-- des soies dans la rangée frontale : g. Coptopsylla.

Pour le genre Coptopsylla, les mâles :

- 1 soie dans la rangée frontale, soie acétabulaire se projetant au-dessus de l'acetabulum :

Ce groupe comprend C. mesghalii Farhang-Azad, 1966, qui n'a pas de sinus, C. iranica Farhang-Azad, 1966, C. afghana Lewis, 1973, C. janiceae Lewis, 1973 qui sont toutes trois très proches et se différencient par la forme des deux lobes du basimère séparés par un sinus peu profond.

C. o. olgae Argyropoulo, 1946, C. o. wachschi Labunets et Kafarskaya, 1961 (dont $C$. mesghalii est extrêmement proche) et $C$. trigona Ioff, 1946 appartiennent probablement à ce groupe (nous ne connaissons pas leur sétation céphalique).

Toutes ces espèces présentent un télomère long, arqué, à apex pointu et recourbé, l'extrémité du bras distal du sternite IX est arrondie. 
- 2 soies dans la rangée frontale, soie acétabulaire se projetant au-dessus de l'acetabulum :

Ce groupe comprend C. l. lamellifer (Wagner, 1895) qui présente la particularité d'avoir perdu la soie acétabulaire, C. l. ardua J. et R., 1915, C. l. dubinini Ioff, 1950 et $C$. l. rostrata Ioff et Tiflov, 1934, ces trois sous-espèces possédant la soie acétabulaire comme il est normal. Toutes montrent un sinus profond, un télomère long, arqué, à apex pointu et recourbé et un bras distal du sternite IX à extrémité arrondie.

C. arax Isayeva-Gurvich, 1950 et $C$. bondari Ioff, 1946 dont nous ne connaissons pas la sétation céphalique, se rattachent sans doute à ce groupe.

- 2 soies dans la rangée frontale, soie acétabulaire se projetant au niveau de l'acetabulum :

C'est le groupe "bairamaliensis ». Il comprend C. neronovi Farhang-Azad, 1972, qui ne possède pas de sinus, $C$. africana Wagner, 1932 qui montre une petite incisure, C. bairamaliensis Wagner, 1928 et C. barbarae Lewis, 1973 (qui est peutêtre un synonyme de la précédente, tout au plus une sous-espèce) qui présentent une incisure plus profonde et $C$. mofidii Farhang-Azad, 1966 qui a un véritable sinus.

C. smiti Hubbard, 1956, dont nous ne connaissons pas la sétation céphalique appartient probablement à ce groupe ; $C$. mofidii en est très proche.

Toutes ces espèces ont un télomère trapu, à bord antérieur droit et à bord postérieur convexe formant un angle aigu et le bras distal du sternite IX triangulaire à apex pointu.

Nous ne pouvons classer ni $C$. caucasica Isayeva-Gurvich, 1950 , ni C. macrophthalma Ioff, 1950 faute d'éléments de morphologie suffisants.

\section{4 - LES PROBLèMES LIÉS AU PARASITISME PAR LES TYLENCHIDES (NEMATODEA)}

Le parasitisme par les Tylenchides est particulièrement fréquent chez les Coptopsyllidae (Beaucournu et coll., 1987).

Après traitement à la potasse en vue du montage, il devient impossible de mettre en évidence la présence de Tylenchides dans les puces. Ce parasitisme peut donc passer inaperçu, ce qui n'est pas sans conséquences pour le taxonomiste car ces nématodes induisent chez les mâles de Coptopsyllidae des malformations, en particulier du segment IX, qui pourraient être interprétées comme des caractères normaux attribués à un taxa nouveau. Ainsi $C$. lamellifer ardua J. et R., 1915 n'a-t-elle pas été décrite d'après un mâle holotype "castré " (Hopkins et Rothschild, 1956) qui diffère sensiblement d'exemplaires normaux au niveau du segment IX.

Les malformations peuvent être très subtiles et se répéter identiquement pour plusieurs spécimens : nous avons ainsi en collection plusieurs mâles de Neocoptopsylla wassiliewi, parasité par des Tylenchides, qui présentent la même morphologie aberrante du tergite IX (fig. 18). 
Il convient par conséquent d'être très vigilant à l'égard de spécimens isolés de Coptopsyllidae et de vérifier préalablement qu'ils n'étaient pas parasités par des Nématodes avant d'interpréter toute morphologie nouvelle.

\section{BIBLIOGRAPHIE}

Beaucournu J.-C., Bach-Hamba D., launay H., Hellal H., Chastel C. : Contribution à l'étude des Siphonaptères de Tunisie. Bull. Soc. Sci. Nat. Tunisie, 1981, 16, 3-9.

Beaucournu J.-C., Hellal H. : Liste annotée des Siphonaptères de Tunisie. Bull. Soc. Pathol. Exot., 1977, 70, 524-537.

Beaucournu J.-C., Kowalski K. : Données nouvelles sur les puces (Insecta, Siphonaptera) d'Algérie. Bull. Soc. Pathol. Exot., 1985, 78, 378-392.

Beaucournu J. C., Launay H., Sklair A. : Les anomalies des spermathèques et des conduits génitaux chez les Siphonaptères (Insecla). Revue bibliographique et cas personnels. Bull. Soc. Entomol. Fr., 1987, sous presse.

Cooneman J. : Siphonaptera recueillis en Libye par la mission X. Missonne (1972-1973). Bull. Inst. Nat. Sci. Nat. Belg., 1973, 49, 1-11.

Hopkins G. H. E. : Notes on synonymy in Siphonaptera. J. Wash. Acad. Sci., 1952, 42, 363-365.

Hopkins G. H. E., Rothschild M. : An illustrated calalogue of the Rothschild collection of fleas (Siphonaptera) in the Brilish Museum (Nalural History). Vol. II : Coptopsyllidae, Vermipsyllidae, Stephanocircidae, Ischnopsyllidae, Hypsophtalmidae and Xiphiopsyllidae. British Museum, 1956, 445 p., 1 carte, 32 pl.

Ioff I. G., Tiflov V. E. : Zur Kenntnis der Flöhe Russlands, insbesondere der Gattungen Stenoponia J. \& R., Coptopsylla J. \& R. und Chaetopsylla Kol. Z. Parasitenk., 1934, 7, 362-391.

Jameson E. W., Jr. : Pleioxenous host-restriction in fleas. J. Nat. Hist., 1985, 19, 861-876.

LEwIS R. E. : A collection of fleas (Siphonaptera) from Northern Saudi Arabia. J. Parasitol., $1964 \mathrm{a}, 50,313-318$.

LEwis R. E. : A new species of Coptopsylla Jordan and Rothschild, 1908, from Northern Saudi Arabia with comments and a key to the genus (Siphonaptera: Coptopsyllidae). Proc. $R$. Ent. Soc. Lond. (A), 1964b, 39, 157-165.

LEwIS R. E. : Siphonaptera. Reprinted from International Jordan Expedition 1966 (International Biological Programme. Conservation of terrestrial Communities), 1966, 64-68.

LEwis R. E. : Notes on the geographical distribution and host preferences in the order Siphonaptera. Part 4. Coptopsyllidae, Pygiopsyllidae, Stephanocircidae and Xiphiopsyllidae. J. Med. Ent., 1974, 11, 403-413.

Smit F. G. A. M. : Classification of the Siphonaptera. In: Synopsis and classification of living organisms (Parker S. P., ed.). McGraw-Hill Book Company, New York, vol. 2, 1982, 1232 p., 557-563.

Smit F. G. A. M., Wright L. (= A.) M. : Notes on the Wagner colleclion of Siphonaptera in the Zoologisches Museum, Hamburg. Mitl. Hamb. Zool. Mus. Inst., 1965, 62, 1-54.

Theodon O., Costa M. : A survey of the parasites of wild mammals and birds, in Israel: part one. Ectoparasites. The Israel Academy od Sciences and Humanities, Jerusalem, 1967, $126 \mathrm{p}$.

Wagner J. : Die Bedleutung der Flöhe für die Frage nach der Genesis der Säugetierfauna. Zoogeographica, 1932a, 1, 263-268.

WAGNER J. : Neocoptopsylla wassiliewi g. n., sp. n. und Coptopsylla africana Wagn. Konowia, $1932 \mathrm{~b}, \mathrm{i1}, 168-175$.

Wagner J., WassiliefF A. : Tableaux analytiques pour la détermination des puces rencontrées en Algérie et Tunisie (Manuel pour les médecins étudiant les maladies infectieuses des mammifères). Arch. Inst. Pasteur Tunis, 1932, 21, 431-467.

Wassilieff A. : Les rongeurs et les puces de la Tunisie et leur rôle dans la propagation de la peste. II. Révision des rongeurs de Tunisie. III. Révision des puces. Arch. Inst. Pasteur Tunis, 1932, 21, 298-340. 